\title{
Approaches to knowledge-transfer systems
}

\author{
Oleg P. Pilipenko* and Helena Komissarova** \\ * Odessa State University, Ukraine \\ ** Kiev Institute of Psychology, Ukraine
}

The use of computers for knowledge transfer does not result automatically in success: the efficiency of the process depends on the approach to instruction underlying the knowledge-transfer system in question. In this paper, the authors describe and compare computer technologies used for instruction in Computer-Aided Instruction (CAI) courses, electronic books, and knowledge-transfer systems based on heterogeneous information. They also deal with some psychological issues involved in dialogue, with the adaptability of knowledge-transfer systems, and with psychologically-based computerized instruction.

\section{Introduction}

The use of computer technology in instruction is based on known approaches to instruction, and also promotes new approaches. Contemporary problems in instruction tend to be determined by intensive information-flow and the short timescale of the relevance of information.

\section{Computer-aided instruction courses}

Historically, the primary method of teaching is the so-called 'Nurnberg funnel' method. This is based on the assumption that knowledge in the teacher's head is linked to some 'correct' structure, and the objective of the teaching is to place facts and relations between them into the heads of a student with minimal distortion. Obviously, this is a simplified approach to teaching, which nevertheless, under certain conditions, provides reasonably good results. However, its effectiveness depends heavily on the personality of the teacher, and it significantly underestimates the student's personality, thus decreasing the possibilities of creative learning.

The introduction of the personal computer caused an exponential growth in the number of knowledge-transfer systems based on this approach, and the possibility of widely disseminating an outstanding teacher seemed to be one of its major advantages. 
Knowledge-transfer systems of this kind are built as restricted models of a human teacher. Traditionally, the teacher determines objectives and the means of instruction, makes decisions about the level of interaction, manages time, and evaluates the student's activity and performance. So, in human-computer dialogue which simulates the dialogue between a teacher and a student, the student is mainly a passive partner and the computer is an active, leading partner. This model often assumes that the learner knows almost nothing about the domain in question, nor about the ways to study it. Under these conditions, the computer imposes the sequence in which the domain is to be studied, and thus determines the skeleton of the knowledge-transfer system. Knowledge-transfer systems of this kind are often called Computer-Aided Instruction (CAI) courses.

Two major obstacles are encountered in implementing and using CAI courses. Many people use computers as tools in their everyday work, where the computer is considered a passive partner in the human-computer interaction. For such people, a contradiction appears when it comes to computer-aided learning: the computer which is thought of as a tool and a slave becomes an instructor and a master. Under such conditions, people may feel uncomfortable because their role in the dialogue, now controlled by a computer, has 'changed (Kommisarova, 1994). Such discomfort may adversely affect students' motivation, as well their emotional state, and may decrease the effectiveness of computeraided learning. The second obstacle is that since learning depends so heavily on the personality of the teacher, it makes sense for one author (or a small group of authors) to design a CAI course. This in itself is a constraint.

\section{Electronic books}

According to current educational theory, direct transfer of knowledge from teacher to student is impossible. Learning is a process involving the juxtaposition of, on the one hand, new facts and theories, and on the other, facts and theories already formed in the student's mind, a process which may result in augmenting or restructuring the latter. Instruction within higher education relies heavily on such a process, and thus independent study, in which students can adapt the procedures associated with knowledge acquisition to their own cognitive processes, is encouraged. This approach is also effectively supported by modern technology in the form of electronic books which can be used to deliver information not only as text but also as images, sound and animation (see Barker, 1991), with hypermedia links.

Knowledge-transfer systems of the above-mentioned type use a different model of human-computer interaction. Here, the computer is considered to be a tool which immediately appears comfortable to use although the activity may be new. But there is a clear difference between using the computer as a tool in an everyday activity and as a tool for learning. The field to be studied and its internal structure may be terra incognita for the user. Tables of contents and indexes do little to help a student who does not know the relevant terminology, nor how to begin. The student needs guidelines for an efficient acquisition of knowledge, and additional information about the logical structure of the knowledge-transfer system, as well as about the logical structure of the field in general and the potential links between information 'frames' within the knowledge-transfer system. The feeling of being an independent explorer of a knowledge-domain can increase 
the motivation of a student, but he or she can easily be distracted and simply go off course (Barker, 1990). This can prevent effective knowledge transfer and evoke a negative emotional attitude to the computer, or at least to computer-assisted learning.

We believe that any knowledge-transfer system should incorporate both the knowledge and the techniques needed to use it. The teacher's knowledge about the sequence in which a student should ideally approach the information is of particular importance, and the student also needs to know why certain parts of it are more important than others. Thus categorization of the links between information frames should be an essential feature of the above-mentioned kinds of knowledge-transfer systems. For example, we may specify links which describe a recommended way to learn the proposed material, or several different ways recommended by different teachers, or determined by different learning objectives. Links to information frames relevant to the study of the current frame, and links between frames exploiting the same methodological approach, might also be assigned to particular categories of links.

\section{Adding power to a knowledge-transfer system by means of dialogue}

Dialogue can be used as a method of access to data contained in knowledge-transfer system. The dialogue scenario should generate information about why and how one should use the system. Some authors of electronic books offer a sequence of questions and answers. In such cases, the roles of the participants are strictly determined: one is the active partner, the other is the passive partner, though this situation can be reversed - the answering (passive) partner can momentarily take the initiative if he or she does not have enough information to answer the question. The answering partner may also take the initiative when the asking (active) partner fails to put the question in a way that is clearly understandable by the answering partner, and the latter may need to ask a series of questions in order to determine the precise demands of the asking partner. The changing of roles in partner dialogues of the kind under consideration here are discussed in more detail in Pilipenko (1994).

We believe that a reasonable balance between activeness and passiveness in such dialogues is vital for effective learning to take place when using knowledge-transfer systems. At each step of the human-computer dialogue, the system should provide the user with all necessary information, including information about its own built-in functionality. Students feel comfortable if they feel they are in control, and if they can predict the overall behaviour of the knowledge-transfer system.

\section{Knowledge-transfer systems delivering heterogeneous information}

Electronic books lend themselves well to situations where information from different sources needs to be presented in a homogeneous form, though it is then important to explain the connections and differences between the viewpoints, and perhaps the terminology, adopted by different authors. However, a particularly interesting trend, based on the idea of the usage of heterogeneous information in knowledge-transfer systems, is that of so-called open hypermedia systems. A significant development related 
to this approach is the Microcosm system developed at Southampton University (Davis et al, 1992). The Microcosm environment allows information frames created with different applications to be incorporated into one system. Markup information, which is used to designate links between information frames, is physically separated from corresponding frames. Thus the documents included in the knowledge-transfer system are always available for modification within the applications which were used to create them. Computer-based knowledge-transfer systems can thus be modified dynamically, and easily extended not only with new information frames, but also with new links. Knowledge-transfer systems based on this approach can support several methods of structuring information simultaneously, and along with heterogeneous information frames, can contain a heterogeneous system of links, for example recommendations as to how and why to access information frames composed by different teachers or intended for students with different backgrounds or different objectives. Learning supported with knowledge-transfer systems based on open hypermedia can also be underpinned by dynamic modifications made by a teacher while using the system, as he or she finds new links which can then be added: learning by doing is thus especially suited to such systems. New links, and possibly new information frames, can be created as a direct result of the search for information relevant to the problem in hand.

\section{Shared computer books}

Another way to use the unique potential of dynamically modifiable knowledge transfersystems lies in the idea of shared electronic books, that is, made available to several users simultaneously. Shared electronic books can be open not only for joint access, but also for modification by all users. Users can add new facts, new ways of handling facts already contained in the systems, and comments. A shared electronic book can become the joint creative output of many people.

The potential of the World Wide Web is also interesting in terms of the development of shared electronic books. The most interesting feature of the World Wide Web is that there is no centralized maintenance or support. It is the result of the creativity of many people. On the other hand, the very absence of centralized support means that the Web cannot perform the role of active partner in a human-computer dialogue: the information contained in the Web is poorly structured (and searching for information can at present take an unacceptably long time). Nevertheless, we believe that information has the strange ability to be self-structuring, a property which can manifest itself both in the intentional actions of a co-ordinated group of people, and in the chaotic activity of different people who do not even know of the existence of each other. We therefore expect that the increasing amount of information available on the Web will not lead to its collapse, as many would argue, but rather to an improvement of its structure.

\section{Adaptability of knowledge-transfer systems}

Knowledge-transfer systems should ideally be able to adapt to student needs, including taking into. account different personal styles of knowledge acquisition. Knowledgetransfer systems should also keep track of the dialogue between user and computer, and memorize which information frames have been accessed by the student, as well as 
evaluating the student's progress and level of comprehension of the material placed in the system. Monitoring dialogue traces is particularly important, since the student who is just starting to tackle a new subject can then have access to the ways in which it has been tackled by other students. In addition, the teacher (or group of teachers) responsible for the maintenance of the knowledge-transfer system can make appropriate corrections to the dialogue scenario if most the students are not using it efficiently (at critical points students may have to be guided more actively).

\section{Conclusion}

The ultimate degree of adaptability in instruction is complete individualization. To achieve this, a computer-based knowledge-transfer system would have to take into account not only the intellectual background and progress of a student, nor only errors and general and specific performance, but it would also have to adopt a style of instruction precisely fitting the learning style of the student, including his or her cognitive and personality/psychological features such as holism/serialism, impulsivity/reflexivity, flexibility/rigidness, verbal/image thinking, introversion/extroversion, field-dependence, level of anxiety, and so forth. Such a task would require a thorough probing of a student's personal characteristics with a series of psychological tests, and careful monitoring of his or her learning activity and performance.

We do not foresee such knowledge-transfer systems in the near future. At present, only the first of the four accepted divisions of computer-based instructional systems (domain or field knowledge-base, pedagogical module, student module, and interface) have been developed to a satisfactory degree. Theoretical and technical progress in interface construction is significantly poorer. The same applies to the pedagogical division, and the problems of student-module design are not even yet solved in principle. Nevertheless, while waiting for progress in these areas, we should pay as much attention as possible when designing knowledge-transfer systems to individualization, and to the issues related to the dialogue between human being and computer.

\section{Editorial note}

This paper was given at a NATO ASI (Advanced Studies Institute) held in Heriot-Watt University in the summer of 1994.

\section{Acknowledgements}

We should like to thank Professor Ray McAleese and the British Council for their assistance and support.

\section{References}

Kommisarova, H. (1994), 'Psychological peculiarities of man-machine communication in instructional systems' in: Brouwer-Janse, M.D. and T. L. Harrington T.L. (eds), HumanMachine Communication for Educational Systems Design, NATO ASI Series, vol. F129, Berlin, Springer-Verlag. 
Barker, P. (1990) 'Designing interactive learning systems', Educational and Training Technology International, 27 (2).

Barker, P. (1991) 'Electronic books', Educational and Training Technology International, 28 (4).

Pilipenko, O.P. (1994), 'Modeling a partner in a dialogue' in Brouwer-Janse, M.D. and Harrington, T.L. (eds), Human-Machine Communication for Educational Systems Design, NATO ASI Series, vol. F129, Berlin, Springer-Verlag.

Davis, H.C., Hall, W., Heath, I., Hill, G. and Wilkins, R. (1992), 'Towards an integrated information environment with open hypermedia systems' in Lucarella, D., Nanard, J., Nanard, M. and Paolini, P. (eds), Proceedings of the ACM Conference on Hypertext: ECHT'92, Milan, ACM. 